\title{
Design and Analysis of a Catamaran Data Acquisition Platform for Water Optics
}

\author{
RUILONG Luo ${ }^{1, a^{*}}$ \\ ${ }^{1}$ Shanghai Ocean University, No. 999, Huchenghuan Rd, Nanhui New City, Shanghai, China \\ arlluo@shou.edu.cn
}

Keywords: Data acquisition platform, Water optics, Catamaran.

\begin{abstract}
The experimental work for water optics cost a lot of time and efforts. Researchers in many places still depend on the traditional lifting sensor methods of measuring and recording water optics data. In this work, a new design of data acquisition platform is described, which is equipped on a platform shaped as a catamaran. The shading effects of platform structure is analyzed which is related to solar zenith and azimuth angle, floating body's dimensions and draft. The main advantage of this platform is its flexibility of altering the type of sensors and ability of remote testing.
\end{abstract}

\section{Introduction}

The quantity and quality of light are critical factors limiting aquatic plant photosynthesis, growth and distribution [1 3]. The underwater light data can be used in vegetation physiology, ecology restoration and remote-sensing retrieval. Time-series photosynthetically available radiation at the surface (surface PAR, 400-700 nm) observations of optical fields near the base of the Western Pacific Subarctic Gyre were conducted from 2005 to 2006 with a Bio-optical Long-term Optical Ocean Measuring System package (BLOOMS). The BLOOMS consisting of a fluorimeter, a spectral radiometer, a depth sensor could record data every $120 \mathrm{~min}$ [4]. Huovinen and Gómez measured the spectral attenuation of solar irradiation in the intertidal and shallow sub tidal in southern Chile to evaluate the underwater light climate's effect to the fjord system [5]. The influence of ecological restoration of Wuli Lake in China was assessed with a parameter of PAR attenuation coefficients [6].

For short term in-situ measurement, researchers conducted the water optics experiments using a hoist lifting the measuring sensors into different depth of water. The hoist was deployed on the ship and controlled by one or two operators. The experiment work cost a lot of time and effort. For constant monitoring, especially for marine environmental monitoring, kinds of mooring buoy were developed and applied [7,8]. These buoys had good stability and performance, but high-cost in build and maintenance.

In this work a remote data acquisition system for measuring underwater PAR data is developed. The system is equipped on a catamaran platform which can be moored to a ship or alongside the bank. The radiation sensor can reach maximum depths of to about $10 \mathrm{~m}$ automatically. Recording data can be transmitted to three kilometers away. The present paper describes the overall structure and function of the system. The instrument suite that is present installed on the platform is introduced in detail, and the shading effects is also analyzed to demonstrate the feasibility and applicability of the new platform. Several modifications for the structure is proposed.

\section{Description of the System}

The system includes five parts: the ground monitoring center, the measurement box, the lifting mechanism, the remote wireless device and the power supply unit. The ground monitoring center is responsible for controlling and monitoring the data acquisition system, receiving and recording the experimental data on personal computer. One operator can start/shut the system and control the depth of the sensors into water. The measurement box is responsible of measuring the sensors voltages, conditioning and amplifying the weak signal. The lifting mechanism is obviously designed to drag the sensor during testing. The remote wireless device is designated to transfer data from test point to 
the ground monitoring center. Finally, the power supply unit is designated to provide the proper voltages to the whole system. All the parts except the first one are attached together on a catamaran, for purposes of discussion, called floating instrument platform as shown in Fig. 1.

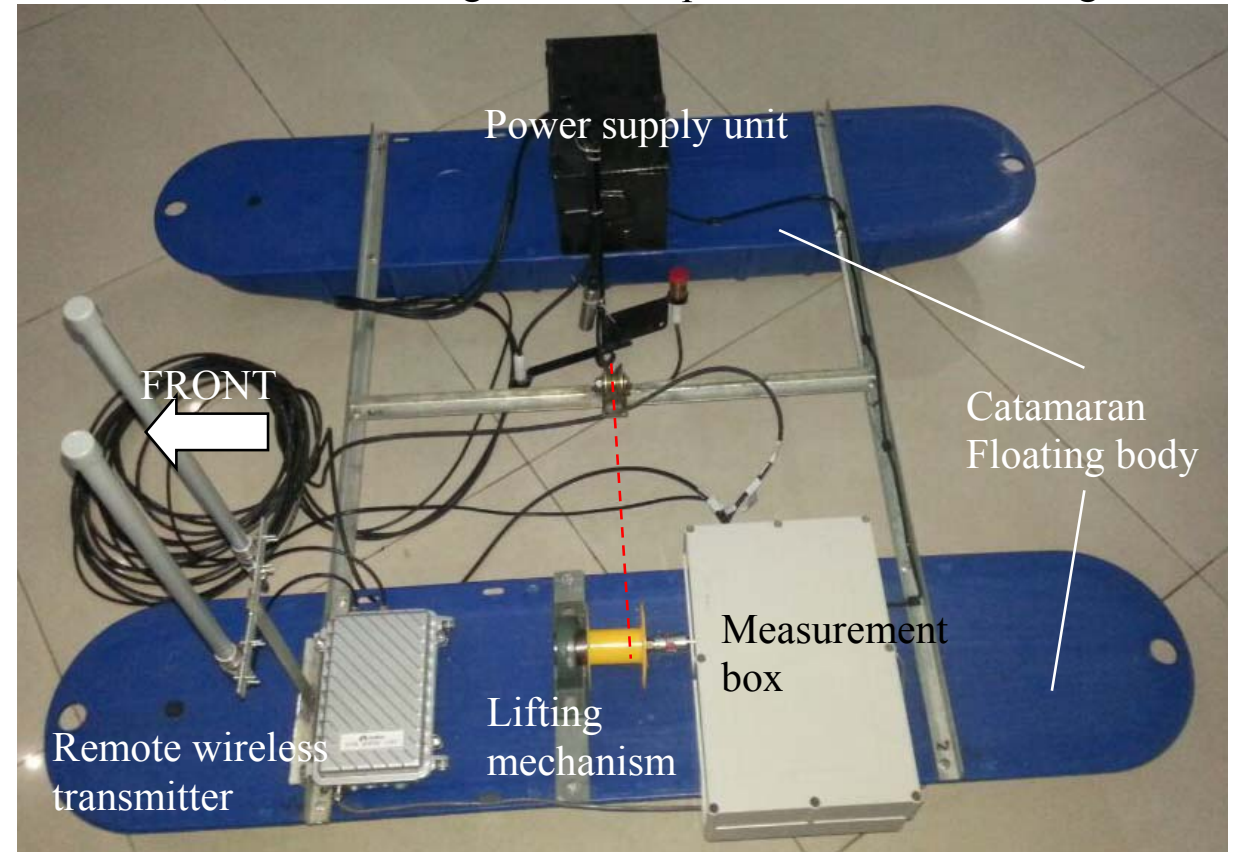

Fig. 1. Photograph of the proposed floating instrument platform.

The catamaran frame structure consists of two separate floating bodies of dimensions $1.60 \mathrm{~m} \times$ $0.32 \mathrm{~m} \times 0.19 \mathrm{~m}$. The floating bodies are connected with two $1.24 \mathrm{~m}$ long angle steel (thickness of $0.05 \mathrm{~m}$ ). Bolt fastening are used to ensure the frame stable and steady. The minimum distance between the floating bodies is $0.6 \mathrm{~m}$ so that there is enough space for the sensor dipping. The floating body is made of polyethylene and hollow which can provide sufficient buoyancy force.

The lifting mechanism comprises the step motor with its driver (placed in the measurement box), the resilient coupling, the hoist drum, the bearing box, the pulley and a nylon rope (shown as the red dotted line in Fig. 1). The bearing box is fastened on one floating body with several bolts. The pulley is welded to the midpoint of a $0.6 \mathrm{~m}$ long angle steel. The step motor drive the hoist drum around which the rope attached to the sensor being lifted and taken over the pulley.

The power supply unit is used to provide the lifting mechanism with $48 \mathrm{~V}$ DC voltage as shown in Fig. 1. This unit consist of four lead acid storage batteries with $12 \mathrm{~V} \mathrm{DC}$ voltages that are connected in series. At present, the system can non-stop run for 20 hours, and a solar panel with charger is under consideration.

The remote wireless equipment includes the transmitter and the receiver. The transmitter with a pair of Omni antennas is placed alongside the measurement box while the receiver with a unidirectional antenna is placed near the ground monitoring center. This allows the operator can set up the monitoring center anywhere suitable within three kilometers around theoretically. To minimize the obstacle (e.g. buildings, trees, or structures) is a prime consideration.

Fig. 2 (a) shows the construction details of the measurement box, which consists of two main parts: (1) the EM9636BD and (5) the step motor. The EM9636BD is a multi-function high accuracy data acquisition (DAQ) device from ZTIC Company (Beijing, China) with an Ethernet connector of RJ45, making it easy to connect to the remote wireless transmitter without any adapter. The resolution is 16 bits with an error of less than $\sim 0.02 \%$. Also, it has a reasonable number of Analog input/Digital output channel (32 AI channels/16 DO channels) for signal gathering and switch controlling. Among them, three AD channels have already been occupied in the current system, which means it still has the capability to increase the number of sensors (e.g. temperature, $\mathrm{pH}$, salinity et al.).

The real-time PAR signal is very weak, approximately $0-8 \mathrm{mV}$, usually less than $\sim 4 \mathrm{mV}$. It is a proper way to amplify the original signal to output of 0-5 V first by (2) the condition module, and then access to the AD channel. Another function is to isolate and remove the noise from sensors. 
The lithium battery (3) with capacity of $160 \mathrm{Ah}$ is used to provide the DAQ device, the condition module and the remote wireless transmitter with $12 \mathrm{~V} \mathrm{DC}$ voltage as shown in Fig. 1 and Fig. 2 (a). The power of the DAQ device and the condition module are $3.6 \mathrm{~W}$ and $1 \mathrm{~W}$, respectively. And hence the continuous operating time can reach up to 421 hours (17 days) theoretically. Besides, the field experiment will cost only tens of minutes in one point. So that the operating time will continue longer. In view of this, no extra battery charging circuit is prepared temporarily.

The 86BYG hybrid step motor (model No. 34IP290-03, Changzhou Prostepper Co., Ltd., Jiangsu, China) is used to tow (6) the hoist drum and lift the sensors. Compared with other similar products, it has much high water-proofing property of IP65. Two DO channels are designated to control motor start/stop and rotation direction. The DAQ device outputs Pulse Wavelength Modulation (PWM) waveform to adjust the motor rotational speed.

Fig. 2 (b) shows two different kinds of sensors: $(8,9)$ the LI-192SA underwater PAR quantum sensors and (10) the PTJ301 immersion depth sensor. The LI-192SA sensor (diameter of $3.18 \mathrm{~cm}$, height of $4.62 \mathrm{~cm}$ ) is product of LI-COR, Inc. (Lincoln, Nebraska, United States), which is known for its high accuracy (maximum deviation of $1 \%$ up to $10,000 \mu \mathrm{mol} \mathrm{s}^{-1} \mathrm{~m}^{-2}$.). It has been cosine corrected and features corrosion resistant, rugged constructions for use in freshwater or saltwater and pressure up to $5500 \mathrm{kPa}$ (560 $\mathrm{m}$ depth) [9]. Two PAR sensor are placed on (11) the 2009S lowering frame, one each for down-welling or upwelling radiation shown as Fig. 2 (b). The frame made of anodized aluminum provides stability for proper orientation of the sensors and minimizes the shading effects. Extra weight can be attached on the lower mounting ring for stabilizing if necessary [9]. The PTJ301 immersion depth sensor bound together with the frame has a measurement range of $10 \mathrm{~m} \mathrm{depth}$. The power of the sensor is provided by the lithium battery with $12 \mathrm{~V} \mathrm{DC}$ voltage simultaneously.
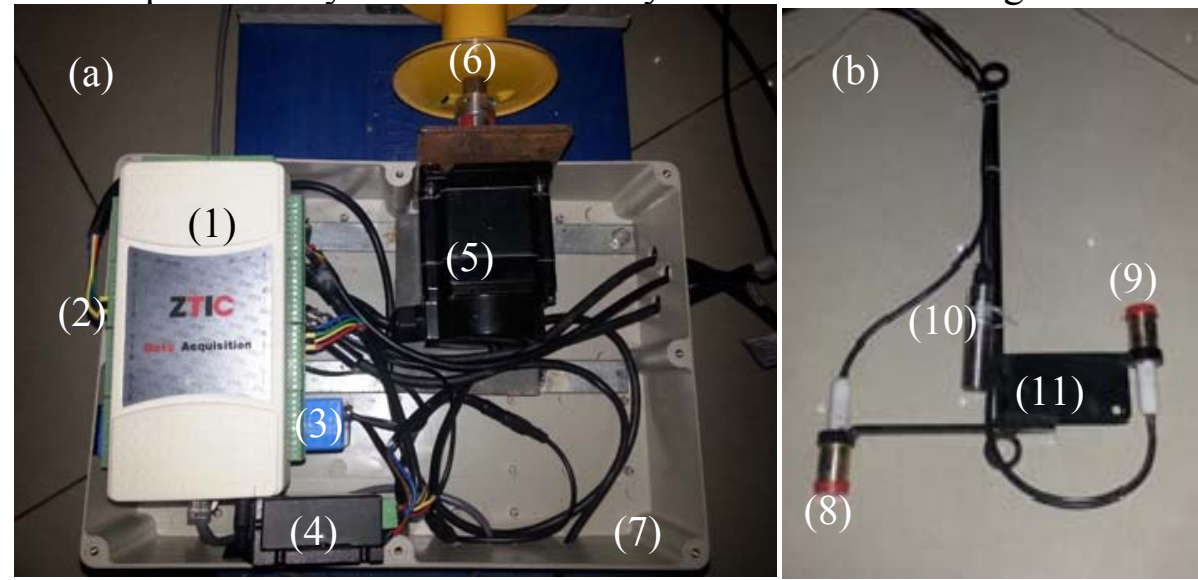

Fig. 2. Photograph of (a) the inner parts of the measurement box and (b) the sensors.

(1) DAQ device; (2) Signal condition module (below the DAQ device, not shown); (3) Lithium battery; (4) Step motor driver; (5) Step motor; (6) Hoist drum; (7) Waterproof case; (8) Upwelling PAR sensor; (9) Down-welling PAR sensor; (10) Immersion depth sensor; (11) Lowering frame.

\section{Shade effect Analysis}

In the system, the lowering frame for sensors placement is as thin as possible to avoid generating self-shade, so that the underwater part's effect on the light field is negligible and the main source of shade effect is the floating body at the water surface. The calculation shows that the measurement error would reach up to $20 \%$ when the solar incident beam enters from the floating body side. Conversely, the error would drop to about $1 \%$ when the beam enters from the sensor side [8]. Therefore it is necessary to assess the floating body's shading effect.

As shown in Fig. 3, two limiting conditions of the floating body are considered: (a) twirling and following the incident beam, and (b) remaining rest and facing north, where the value $\theta_{1}$ is the zenith angle; $l$ is the minimum distance between the test point $O$ and the floating body; $\varphi$ is the azimuth angle (west is zero); $P$ is the midpoint of inner side of floating body; $Q$ is the intersection of extension 
line of incident beam projection and inner side of floating body, relating to time or $\varphi$. The maximum of $\overline{O Q}$ is $0.85 \mathrm{~m}$.

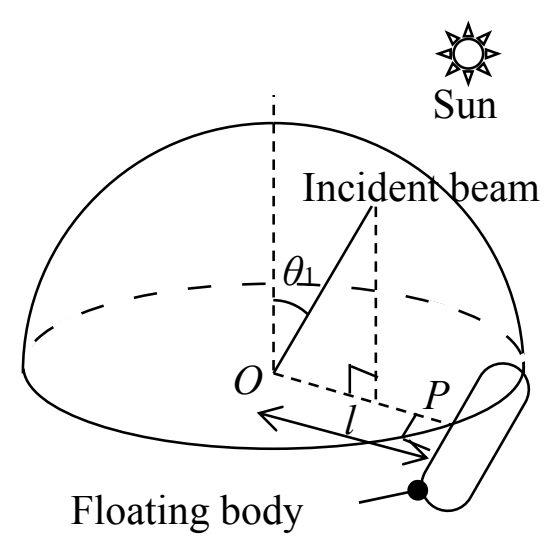

(a)

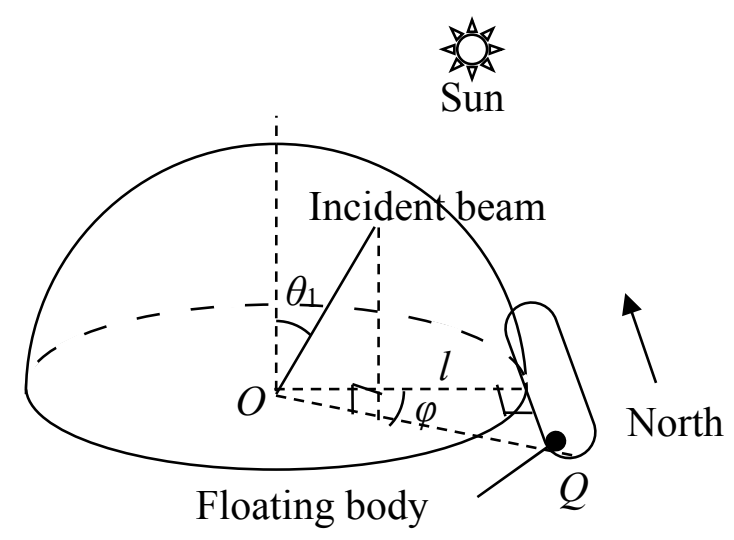

(b)

Fig. 3. Schematic of both of limiting conditions of the floating body: (a) twirling and following the incident beam, and (b) remaining rest and facing north.

The condition (a) is analyzed first. Assume that the cross-sectional areas of the floating body is a rectangular with dimension of $h$ height by $w$ width. The height of the surface is $h_{1} . \theta_{1}$ and $\theta_{2}$ are the angles of incidence and refraction, respectively. $l_{1}$ is the distance between the beam incident point and the inner side of the floating body. The shadow area appears because of light intercepted as shown in Fig. 4. The sensor dips at the point $O$ with the lowering frame. It sequentially experiences three stages: normal, shadow and normal as with area A, B, and C shown in Fig. 4, respectively. The depth $d_{1}$ is the length from the water surface to the top boundary of the shadow area. The larger the depth is, the smaller the error is. The depth $d_{2}$ is the length of the shadow area.

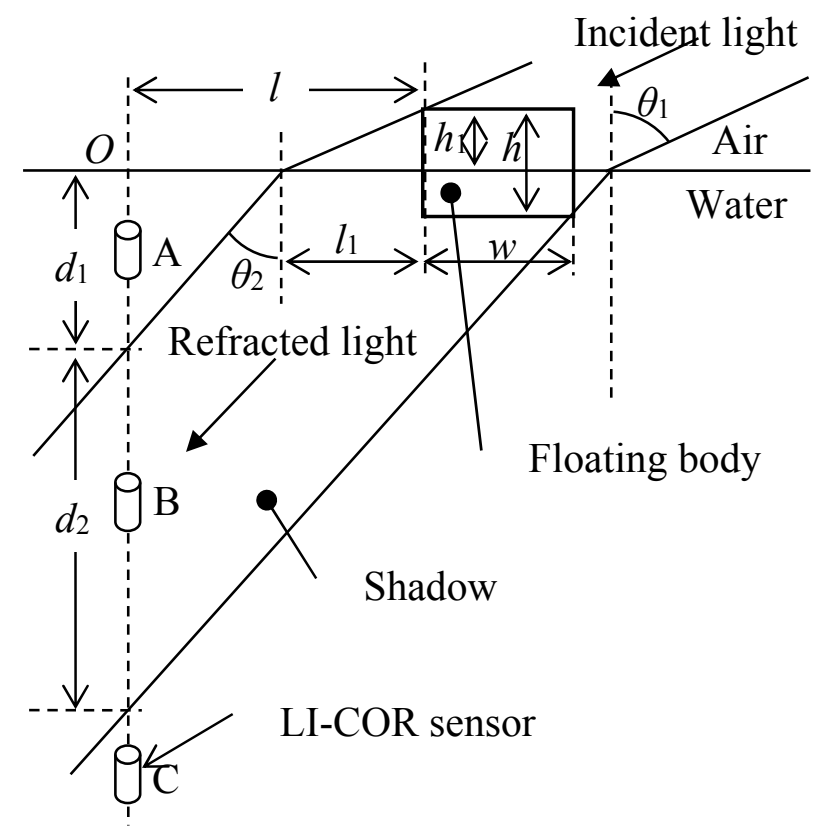

Fig. 4. Schematic of the shading effect because of the floating body.

When the incident angle $\theta_{1}$ is known, the refracted angle $\theta_{2}$ can be calculated from Snell's law:

$$
\mathrm{n}_{21}=\sin \theta_{1} / \sin \theta_{2}
$$

where $\mathrm{n}_{21}$ is the refractive index. In the current work, it is 1.333 . When the floating body is determined, the width $w$ and the height $h$ are constant value. After that, the parameter of the depth $d_{1}$ and $d_{2}$ can be defined as: 


$$
\begin{aligned}
& d_{1}=\left(l-h_{1} \tan \theta_{1}\right) / \tan \theta_{2}, \\
& d_{2}=\left(h_{1} \tan \theta_{1}+h\right) / \tan \theta_{2}+w-h_{1} .
\end{aligned}
$$

Obviously, with the exception of the parameters of $\theta_{1}$ and $\theta_{2}$, the depth $d_{1}$ is related with the surface height of the floating body $h_{1}$ and the inner space of the structure $l$, while the depth $d_{2}$ depends on $h_{1}$ height only. Fig. 5 shows that the height $h_{1}$ has no significant influence on both $d_{1}$ and $d_{2}$. When the zenith angle $\theta_{1}$ is less than $10^{\circ}$ (around noon, the water optical testing time), the depth $d_{1}$ is $2 \sim 4 \mathrm{~m}$ and the depth $d_{2}$ is $3 \sim 5 \mathrm{~m}(l=0.30 \mathrm{~m})$. It represents that the experiment data get large error in nearly full-range which is unacceptable. With the zenith angle $\theta_{1}$ increases, the depth $d_{1}$ decreasing rate slows down. When the angle $\theta_{1}$ is approximately $60^{\circ}$ [see Fig. 5 (a), the blue triangle], the depth $d_{1}$ is zero even minus value, which means the sensor stays in the shadow area since water surface. The decreasing trend of the depth $d_{2}$ looks like $d_{1}$ and dropped to $1 \mathrm{~m}$ depth [see Fig. 5 (b)]. When the angle $\theta_{1}$ is larger than $60^{\circ}$, the shadow area $d_{2}$ has a marked increase. Besides, the larger the angle $\theta_{1}$ is, the more marked it becomes. In contrast, the length $l$ has a major effect on the depth $d_{1}$. When $l$ increases to $1.00 \mathrm{~m}$, the depth $d_{1}$ reaches above and beyond $10 \mathrm{~m}$ [see Fig. 5 (a), the red circle]. The result of Cao's study indicated that, in such cases, the shading error is less than $1 \%$ which can be negligible [8].

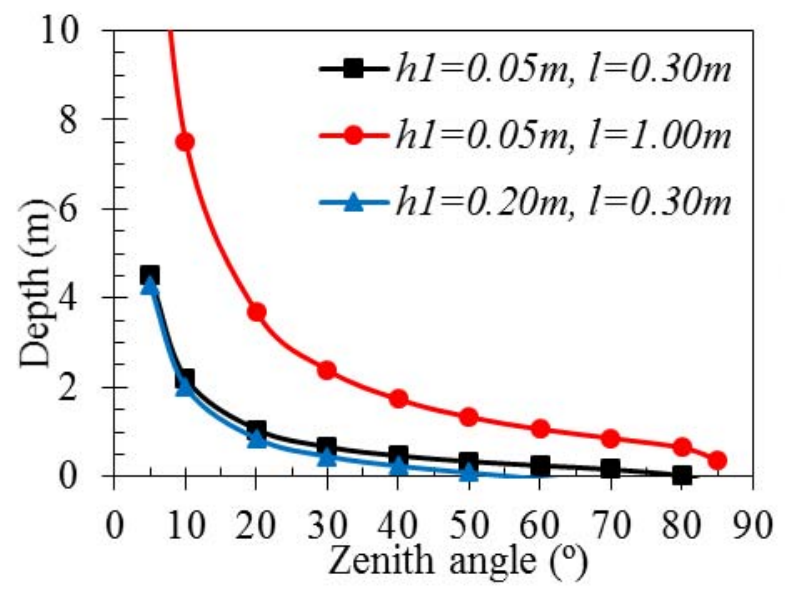

(a)

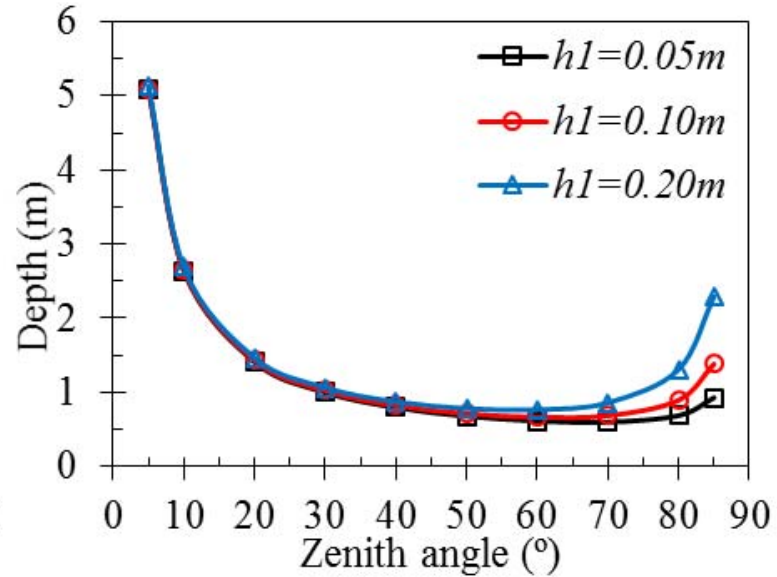

(b)

Fig. 5. (a) Comparison of the depth $d_{1}$ for $h_{1}$ of $0.05 \mathrm{~m}$ and $0.20 \mathrm{~m}$, and $l$ of $0.30 \mathrm{~m}$ and $1.00 \mathrm{~m}$. (b) Comparison of the depth $d_{2}$ for $h_{1}$ of $0.05 \mathrm{~m}, 0.10 \mathrm{~m}$ and $0.20 \mathrm{~m}$, respectively.

Under the condition (b) shown in Fig. 3, the floating body maintains the state of rest and points at north. It is easy to see that the structure can shade the solar radiation for a period time depending on the azimuth angle $\varphi\left(\varphi \leq 69.5^{\circ}\right)$. Assuming the test was conducted in June 1, 2015 in Shanghai $(\mathrm{N}$ $31.23^{\circ}, \mathrm{E} 121.48^{\circ}$ ), substitute $h_{1}=0.05 \mathrm{~m}, l=\overline{O Q}=0.85 \mathrm{~m}$ and $\varphi=69.5^{\circ}$ in the Eq. 1 and Eq. 2 , and then solve the angle of $\theta_{1}$ is about $10^{\circ}$ and the depth $d_{1}$ is $6.38 \mathrm{~m}$. When $69.5^{\circ} \leq \varphi \leq 110.5^{\circ}$ (around noon), the shade of the structure departs from the sensor and causes mild errors. However, because the test point is in the middle of the frame, the angle $\varphi$ of no shading effect area is greatly curtailed.

As to the above analysis, the system has the ability to conduct the field test of water optics around noon, when the structure maintains rest and faces the north. For the purpose of extending the applicability and duration, the system can be modified in the following areas: (1) increasing the inner space of the catamaran frame structure; (2) moving the test point to rear area or beyond the platform if possible.

\section{Conclusions}

In this paper, the development of water optics data acquisition system is designed and described. The proposed system choses a catamaran as infrastructure for purpose of being stable and steady. Around noon, the system can conduct field test with mild error of less than $1 \%$ theoretically, 
considering the prerequisites of being rest and facing the north. In order to improve the applicability and duration, several modifications are necessary. The present system has the advantages of flexibility of changing sensors and remote measuring.

\section{Acknowledgement}

This work is fanatical supported by the Youth Teacher Training Program of Shanghai Education Commission, the Specialized Funds for Scientific and Technological Development of Shanghai Ocean University (Grant No. A2-0209-14-200065) and the Doctoral Starting up Foundation of Shanghai Ocean University (Grant No. 2013).

\section{References}

[1] F. Arthaud, M. Mousset, D. Vallod, et al, Effect of light stress from phytoplankton on the relationship between aquatic vegetation and the propagule bank in shallow lakes, Freshwater Bio. 4 (2012) 666-675.

[2] D. Deregibus, M.L. Quartino, G.L. Campana, et al, Photosynthetic light requirements and vertical distribution of macroalgae in newly ice-free areas in Potter Cove, South Shetland Islands, Antarctica, Polar Biol. (2015).

[3] B. Qin, Y. Zhang, G. Gao, et al, Key factors affecting lake ecological restoration, Adv. Earth Sci. 9 (2014) 896-906.

[4] M.C. Honda, K. Sasaoka, H. Kawakami, et al, Application of underwater optical data to estimation of primary productivity, Deep Sea Res. Pt. I. 12 (2009) 2281-2292.

[5] P. Huovinen, I. Gómez, Spectral attenuation of solar radiation in Patagonian fjord and coastal waters and implications for algal photobiology, Cont. Shelf Res. 3-4 (2011) 254-259.

[6] Y. Zhang, K. Chen, B. Qin, Study on the improvement of underwater light field in Wuli lake ecological demonstration project, Adv. Water Sci. 1 (2007) 86-94.

[7] D. Antoine, P. Guevel, J.F. Desté, et al, The "BOUSSOLE" buoy-a new transparent-to-swell taut mooring dedicated to marine optics: design, tests, and performance at sea, J. Atmos. and Ocean. Tech. 6 (2008) 968-989.

[8] W. Cao, Y. Yang, J. Zhang, et al, Design and test of moored optical buoy, J. Opt. Technol+. 2 (2010) 1-6.

[9] Information on http://www.licor.com. 